\title{
Squamocolumnar Junction
}

National Cancer Institute

\section{Source}

National Cancer Institute. Squamocolumnar Junction. NCI Thesaurus. Code C97148.

The border between the squamous epithelium that lines the ectocervix and the columnar epithelium that lines the endocervix. 